\title{
The Use of Metal Sublaminar Wires in Modern Growth- Guidance Scoliosis Surgery: A Report of 4 Cases and Literature Review
}

\author{
R. BOGIE, MD, PHD, ${ }^{1,2}$ J.J. ARTS, PHD,${ }^{1}$ S.N. KOOLE, MD,${ }^{1}$ L.W. VAN RHIJN, MD, PHD,${ }^{1}$ P.C. WILLEMS, \\ $\mathrm{MD}, \mathrm{PHD}^{1}$ \\ ${ }^{I}$ Department of Orthopaedic Surgery, CAPHRI School for Public Health and Primary Care, Maastricht University Medical Centre, The Netherlands, \\ ${ }^{2}$ Department of Orthopaedic Surgery, St Anna Hospital, Geldrop, The Netherlands
}

\begin{abstract}
Background: To avoid early fusion and allow residual growth of the spine in early onset scoliosis (EOS) treatment, growth-guided scoliosis surgery can be performed. Four patients with EOS are presented in which a growthguidance instrumentation is used with sliding titanium (Ti) sublaminar cables. Residual growth of the spine can be preserved using metal sublaminar wiring; however, several drawbacks of this technique and type of material are illustrated.

Methods: Four patients with progressive neuromuscular scoliosis were treated with a posterior stabilization. A fusionless growth-guidance instrumentation was used consisting of a combination of lumbar pedicle screws and sliding Ti sublaminar cables along cobalt chrome rods.

Results: In 2 cases, the described growth-guidance technique provided sufficient stability and correction of the curvature with preservation of growth. In 2 patients, the instrumentation failed due to upper thoracic sublaminar wire breakage. The ongoing abrasion of the rod-wire interface caused severe metallosis. In these cases, a debridement and revision surgery was performed with partial fusion of the spine.

Conclusions: Growth-guidance techniques with sliding metal sublaminar wires seem to be a valuable solution for the preservation of spinal growth in EOS surgery. High curvatures, however, have a higher chance of failure and demand for more corrective strength and support of the instrumentation. The use of metal sublaminar wires in a "sliding" instrumentation can lead to early breakage and metallosis.

Level of Evidence: 4, case series.

Clinical Relevance: Surgeons should be aware of possible complications associated with the use of metal laminar wires in spinal fusion and growth-guidance scoliosis surgery. The implementation of materials containing higher fatigue strength and lower friction properties (eg, UHMWPE wires) may avoid these potential complication risks.
\end{abstract}

Complications

Keywords: scoliosis, growth guidance, sublaminar wires, titanium, metallosis

\section{INTRODUCTION}

In the management of early onset scoliosis (EOS), fusion can lead to a short trunk stature with resulting volumetric growth restriction of the thoracic cage, which restricts pulmonary development. ${ }^{1}$ The occurrence of the crankshaft phenomenon with secondary deformity also discourages early spinal fusion. ${ }^{2}$ With the advancement of pedicle screw fixation and with further improvement of sublaminar wiring techniques and mechanical properties, growth-guidance systems are promising techniques to serve as an alternative to spinal fusion in EOS surgery. Examples are the Shilla system ${ }^{3}$ and a modern Luque trolley, ${ }^{4}$ both of which rely on pedicle screws and/or titanium (Ti) sublaminar wires sliding along metal rods. In this manner, partial growth of the spine can be preserved.

Sublaminar wires have been widely used in conjunction with posterior instrumentation in scoliosis surgery. Luque was the first to introduce the Luque-rod segmental spinal instrumentation (LSSI). ${ }^{5}$ LSSI consisted of segmental spinal fixation in which L-rods were attached to the spine using sublaminar wires. However, this type of wiring has some serious drawbacks, such as neurological damage, wire breakage, and difficulties with wire removal. ${ }^{6-11}$ Since the successful introduction of pedicle screw instrumentation in scoliosis surgery, 
today sublaminar wiring is used mainly in hybrid systems. Due to the difficulty of pedicle screw insertion in the deformed spine, sublaminar wiring remains a valuable option to support posterior instrumentation and to realize apical translation of the curve. ${ }^{12,13}$ In case of spinal fusion, the wire must be capable of upholding the corrective load until bony fusion has occurred. If bony fusion has failed, metal fatigue can lead to wire breakage. ${ }^{14,15}$ With the use of sublaminar wires in growth-guidance systems (nonfusion surgery), there is an inherent risk that the high continuous load and abrasion of the wires with the metal rods could increase the chance of instrument failure and subsequent loss of curve correction.

To illustrate the risks and benefits of the use of metal sublaminar wires in a growth-guidance system for the correction of EOS, we present 4 cases in which a modified Luque trolley has been used.

\section{Case 1}

A 10-year-old girl with severe neuromuscular scoliosis (congenital myopathy) presented with a progressive thoraco-lumbar curve (Cobb angle $69^{\circ}$; Figure 1a). Due to curve progression and trunk imbalance, she was indicated for posterior hybrid instrumentation surgery with a modified Luque trolley. In September 2012, a posterior stabilization and correction was performed using a hybrid system consisting of lumbar pedicle screws (L3-L5) and 2 4.75-mm cobalt chrome $(\mathrm{CoCr})$ rods (CD Horizon Solera, Medtronic Sofamor Danek, Memphis, Tennessee) along the curvature. Correction and fixation was achieved by gradually applying tension of Ti sublaminar cables (Atlas cable, Medtronic) at lumbar and thoracic levels (T3-L1). Postoperative radiographic control showed no signs of complications, and correction of the curve was satisfactory (Cobb angle $33^{\circ}$; Figure 1b). After 5 months, routine follow-up radiography revealed fracturing of 5 upper thoracic sublaminar cables with loss of correction of the curve (Cobb angle $44^{\circ}$; Figure 1c). The broken sublaminar Ti cables were surrounded by severe metallosis (Figure 2), for which surgical debridement was performed. Revision surgery was performed with replacement of the proximal wires by hooks, and a spinal fusion at the thoracic level was performed. The postoperative course was uncomplicated, and curve correction was preserved at 3-year radiological follow-up (Cobb angle 27\%; Figure 1d).

\section{Case 2}

In March 2014, a 4-year-old girl with spinal muscle atrophy type 2 and a progressive mild thoracic scoliosis (Cobb angle $29^{\circ}$ ) was scheduled for stabilization surgery, as bracing therapy was not tolerated and progressive sitting balance problems were experienced. The instrumentation consisted of lumbar pedicle screw insertion (L3-L5) and $\mathrm{CoCr}$ rods along the spine fixed with Ti cables at levels T4-L2. At the upper instrumented level, a cross-link was used to enhance stability. After a follow-up of 2 years, no complications had occurred, and integrity of the instrumentation was sustained. Postoperatively, the curve was corrected (Cobb angle $10^{\circ}$ ), and radiographs showed the sliding effect of the sublaminar wires along the rods, which had allowed growth of the thoracic segments (Figure 1b,c). Sitting balance was improved, and a growth of the instrumented levels of approximately $2 \mathrm{~cm}$ was achieved within 2-year follow-up (Cobb angle still $10^{\circ}$ ).

\section{Case 3}

In March 2012, a 7-year-old girl diagnosed with Rett syndrome underwent a posterior spinal stabilization for a progressive thoraco-lumbar scoliosis with a Cobb angle of $67^{\circ}$ (Figure 3a). A hybrid posterior instrumentation with bilateral pedicle screws in L4, L5, and $\mathrm{S} 1$ and $\mathrm{CoCr}$ rods were used. At levels T4-L3, sublaminar Atlas cables were attached to the rods. Curve correction and implant positioning were satisfactory (Cobb angle $44^{\circ}$ ). The postoperative course was uneventful. After 1-year follow-up, the patient complained of back pain without neurological signs or symptoms. The radiographs revealed 2 broken sublaminar cables (level T4 and T5) with resulting loss of curve correction (Figure 4c; Cobb angle $60^{\circ}$ ). During revision surgery, metallosis was observed in the surrounding tissue and debrided at the site of the broken cables. These sublaminar cables were removed and replaced with transverse process hooks, and a spinal fusion was performed at the thoracic levels (Figure 4d). After 4-year follow-up, instrumentation and curve correction were unaffected. As a result of fusion of the spine and placement of the transverse process hooks, no residual growth of the instrumented spine was observed. 

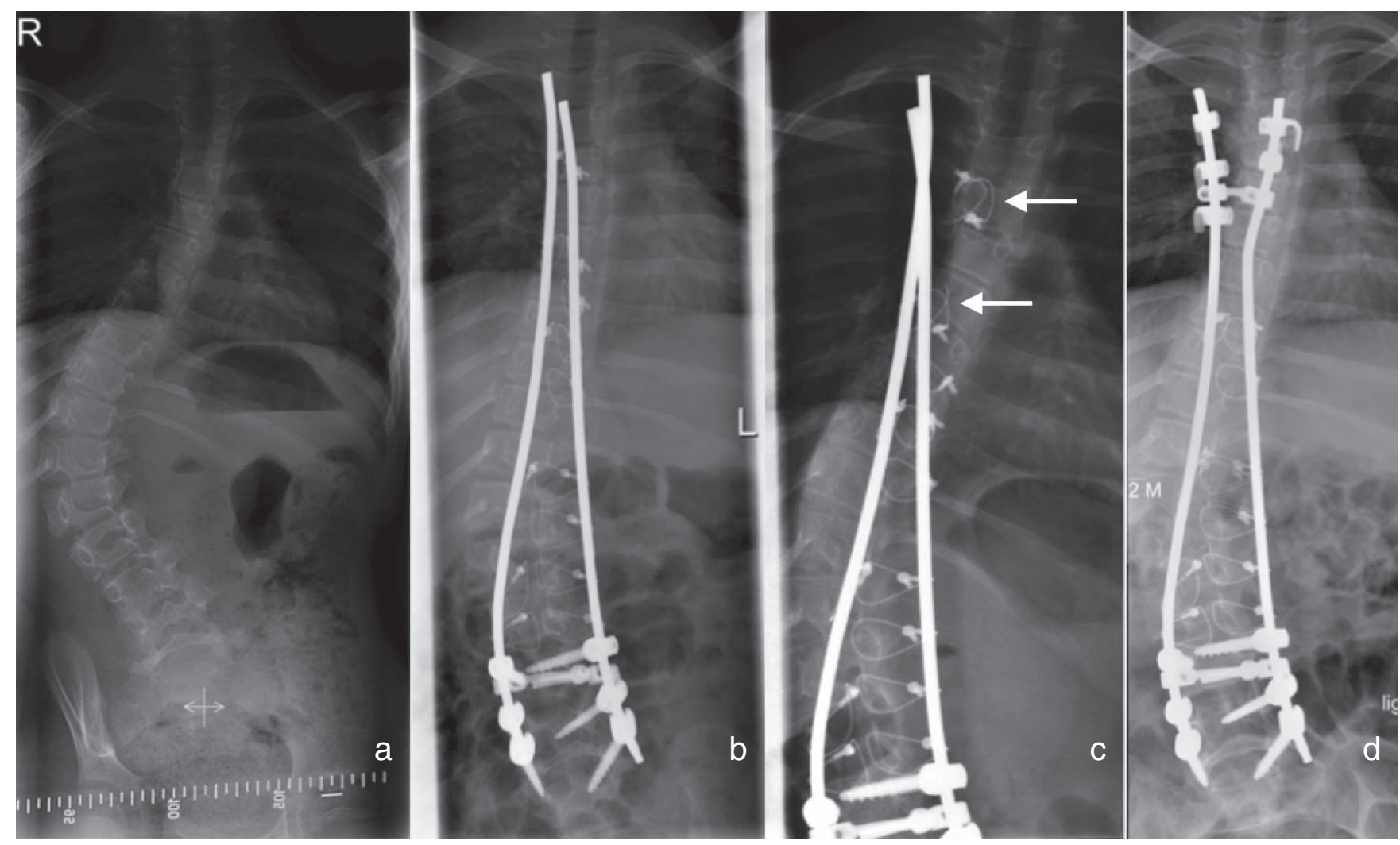

Figure 1. Coronal radiographs. (a) Preoperative scoliosis. (b) Postoperative instrumented spine. (c) Observation of the thoracic broken sublaminar cables (arrows) at 5-month follow-up. (d) 3 years after revision surgery.

\section{Case 4}

In March 2009, a posterior spinal stabilization was performed in a 9-year-old boy with a progressive thoraco-lumbar scoliosis because of spinal muscle atrophy type 1 with chronic respiratory failure for which he used nocturnal noninvasive respiratory support. The combination of a thoracolumbar coronal curve of $24^{\circ}$ with a progressive thoracic kyphosis of $88^{\circ}$ and poor sitting balance in the wheelchair necessitated surgery. A hybrid instrumentation was used consisting of lumbar pedicle screws (L3-L5) and thoracic sublaminar wiring (Th4-L1) fixed to $2 \mathrm{CoCr}$ rods. Postoperatively, the thoraco-lumbar curvature showed a coronal Cobb angle of $14^{\circ}$ and reduction of the kyphosis to $63^{\circ}$ (Figure 5). The postoperative rehabilitation was uncomplicated, and during the last checkup, 6 years postoperatively, there were no complaints, and radiologic evaluation showed preserved instrumentation stability and correction. The growth-guided function of the wire/rod combination permitted a growth of $10 \mathrm{~cm}$ of the instrumented spine in 6 years. This considerable residual growth resulted in sliding of the 2 upper cables out of the rods, which probably contributed to an increase of the kyphosis to $76^{\circ}$ (Figure 5c).

\section{DISCUSSION}

Growth-guidance systems have regained interest in the treatment of early onset scoliosis patients, in which it is imperative to retain longitudinal spine growth, thereby maintaining thoracic cage and lung volume development. ${ }^{16}$ In the reported cases, the instrumentation technique consisted of a modified Luque trolley (an "internal brace" concept) with insertion of lumbar pedicle screws, in which $\mathrm{CoCr}$ rods were stabilized with multistranded Ti sublaminar cables to the thoraco-lumbar spine. Correction of the curve was performed by gradually applying tension to these wires. In 2 reported cases with minor curves (ie, Cobb angles $29^{\circ}$ and $24^{\circ}$ ), the internal brace concept was successful. The sliding effect between the wires and rod in these cases allowed for ongoing growth of the spine. The use of a supportive cross-link in 1 case probably contributed to increased (rotatory) stability. In the 2 other cases, the deformities were more severe (ie, Cobb angles $69^{\circ}$ and $67^{\circ}$ ), and the instrumentation failed. 

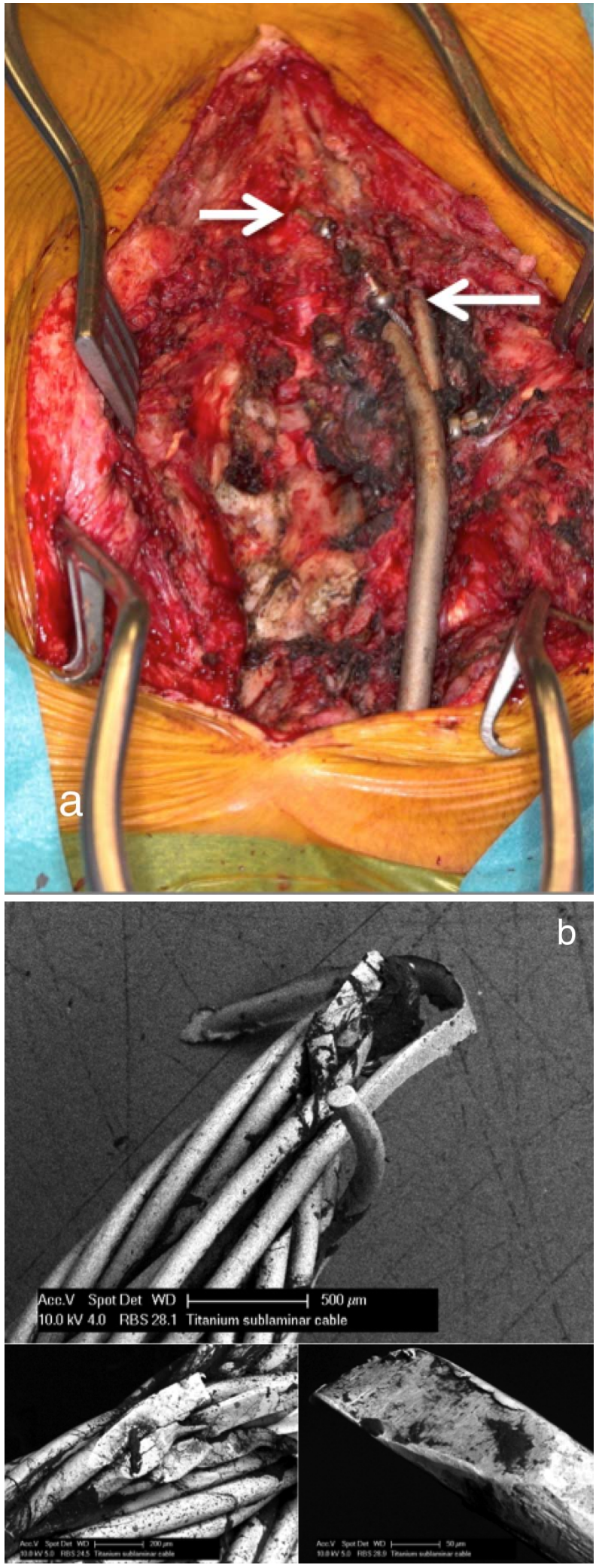

Figure 2. (a) Severe metallosis surrounding the broken titanium (Ti) cables. (arrows). (b) Electron microscopy analysis of a broken Ti cable. The oblique surface of fracture demonstrates signs of metal fatigue failure.
These high curvatures demand more corrective strength and support of the instrumentation and have to withstand high correction and derotation forces. It is likely that in these 2 cases, metal fatigue, as observed during the electron microscopy analysis, eventually led to the breakage of the wires and subsequent instrumentation failure. Ti wear debris caused severe metallosis in the surrounding tissues, which necessitated extensive debridement. In these cases, a revision surgery with fusion of the spine was necessary. Future growth of the spine was thus restricted in these cases.

Multistranded cables replaced monofilament sublaminar wires in the early 1990s. These new cables offered higher static and fatigue strength. ${ }^{17,18}$ This improved wire design with better cable conformance to the shape of the lamina also addressed neurological injury risks. Penetration of the spinal canal seemed less than that of monofilament wire. ${ }^{19}$ Despite the improved wire features, neurological injury can still occur during insertion and removal of the wires. When sublaminar wires are used in growth-guided surgery, the continuous cyclic loading, due to lack of support by, for example, pedicle screws and a bony fused spine, increases the risk of wire breakage. ${ }^{14}$ Ongoing abrasion of the wires against the metal rods can lead to metallosis, as described in this report, which can negatively affect the soft tissue and spinal structures. ${ }^{20,21}$ Spinal metallosis has been described weeks to years after spinal fusion instrumentation in both $\mathrm{Ti}$ (as used in our patients) and stainless-steel constructs. ${ }^{22}$ Recently, metallosis-associated complications with the formation of seroma and sinuses and concentrations of $\mathrm{Ti}$ and vanadium ions in the blood were found in patients after instrumentation of growth guidance sliding instrumentation. ${ }^{23}$ Surgeons should be aware of possible complications associated with the use of metal laminar wires in spinal fusion and growthguidance scoliosis surgery.

An alternative to growth-guidance EOS surgery is the use of growing rods, a distraction-based technique. These implants intend to correct and maintain the spinal deformity via mechanically applying a distractive force across the concavity of the curvature. With the use of the traditionally growing rod techniques, repeated distraction surgery is necessary. Magnetic-controlled growing rods (MCGR) have been developed to avoid these invasive repeated distraction procedures and show similar results in terms of curve control. ${ }^{24,25}$ 


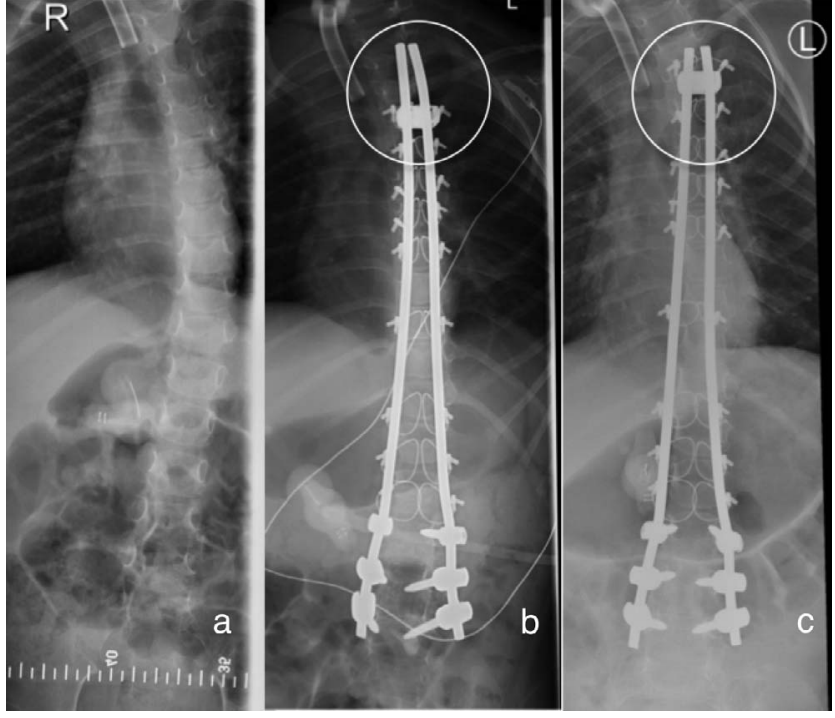

Figure 3. Coronal radiographs. (a) Preoperative curvature. (b) Postoperative instrumentation with correction of the curve. (c) Preserved stability of the instrumentation at 2-year follow-up showing the sliding effect at the most proximal rod/wire juncture (circles). Growth was determined as the difference in length between the proximal rod-cross-link distance 1-day postoperatively and at 2-year follow-up radiography.

Although infection rates are less in MCGR surgery, instrument-related complications are still high with the use of this technique. ${ }^{26}$ Analysis of explanted magnetic expansion control growing rods showed that with the achieved growth during distraction, high volumes of $\mathrm{Ti}$ wear debris were formed. ${ }^{27}$
These findings emphasize the challenge to address metallosis in EOS surgery.

In order to avoid metal wires associated complications, artificial polymeric and polyethylene sublaminar bands have been developed for its use in growth-guidance surgery. The Universal Clamp, using polymeric sublaminar bands as part of a $\mathrm{rod} /$ pedicle screw system, is already used in human corrective spinal fusion scoliosis surgery. ${ }^{28}$ Currently, novel radiopaque ultrahigh-molecular-weight polyethylene sublaminar wires have been tested in a preclinical setting for their use in growth-guidance scoliosis surgery. ${ }^{29}$ The mechanical and biochemical properties of these novel wires can decrease the risk of spinal injury during insertion and removal of the wires while simultaneously attaining higher fatigue strength and low friction. ${ }^{29,30}$ In this manner, metallosis-associated complications could also be addressed and instrumentation failure, as a result of high rotatory and corrective forces, decreased. Simultaneously, a lower friction coefficient will allow for enhanced sliding of the wires along the rod.

\section{CONCLUSIONS}

The described growth-guidance technique in the reported cases seems a valuable option in patients with moderate scoliotic curves in which corrective

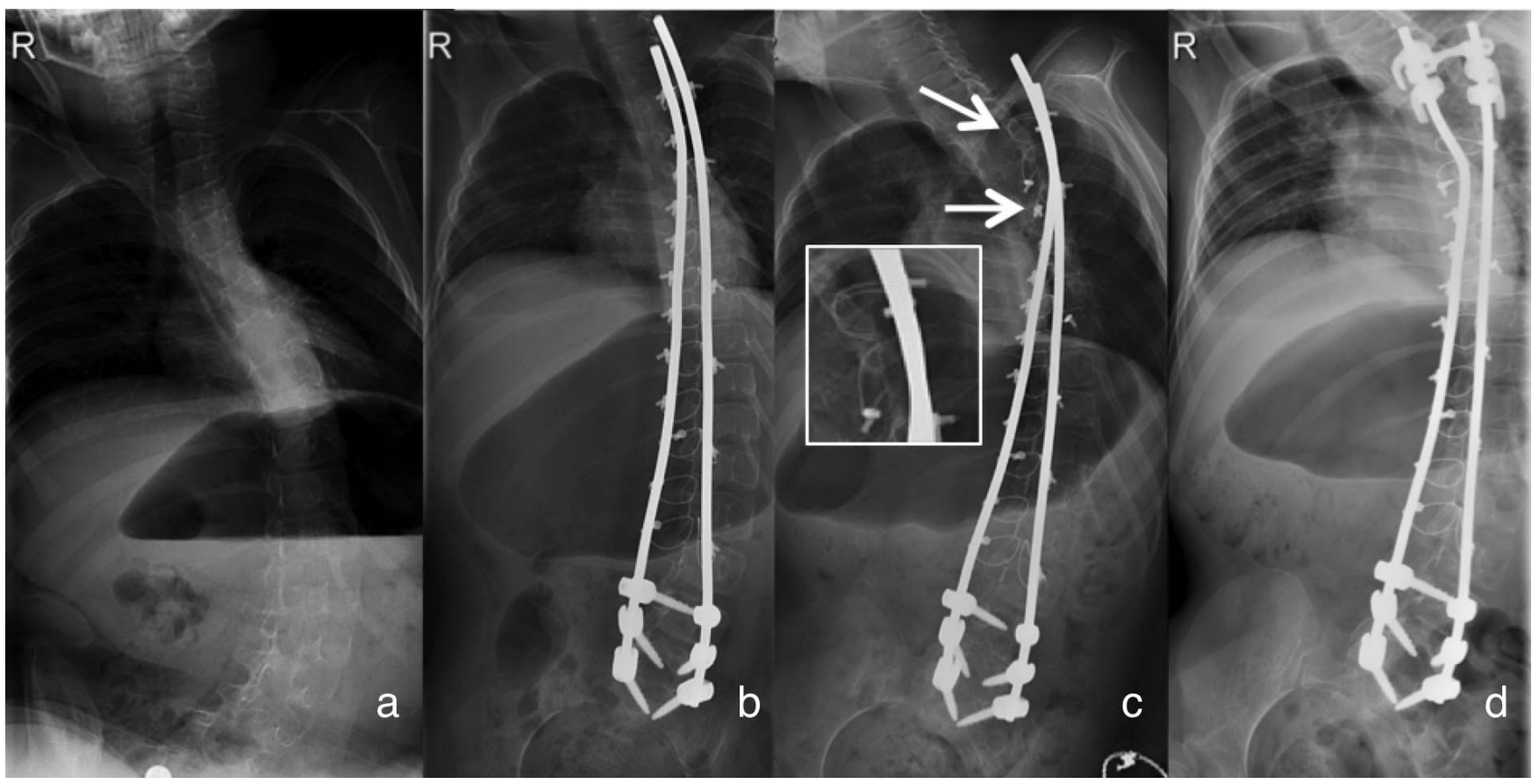

Figure 4. Coronal radiographs. (a) Preoperative curvature of the spine. (b) Postoperative radiograph. (c) Radiograph revealing the 2 broken cables at levels T4 and T5 (arrows). (d) Postoperative radiograph following revision surgery. 

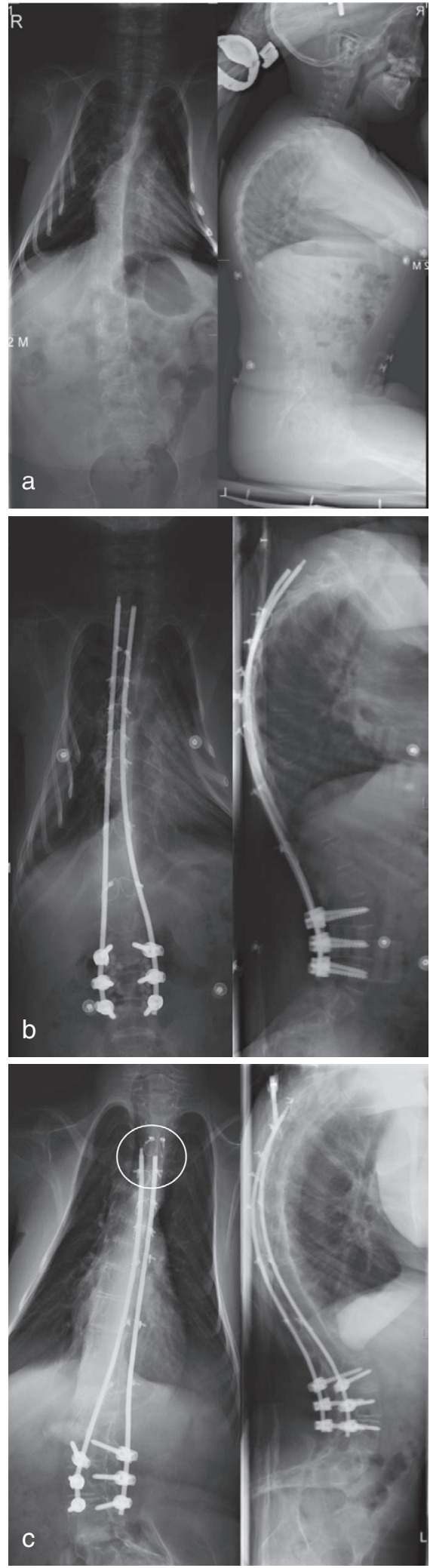

Figure 5. Coronal and sagittal radiographs. (a) Preoperative curvature with a mild frontal Cobb angle and increased upper thoracic kyphosis. (b) Postoperative instrumentation. (c) At 6-year follow-up, the sliding effect facilitated substantial growth. The distal rods have grown out of the T4 sublaminar wire (circle). Growth was measured as the difference in length between the distance of the T4 sublaminar wires and rod ends 1-day postoperatively and at 6-year follow-up radiography. surgery is indicated and preservation of spinal growth is imperative. Improvement of wire characteristics and instrumentation design is still necessary to decrease the risk of complications and increase the stability in correction of more severe scoliosis in growth-guiding scoliosis surgery. The challenge still remains in selecting the timing of procedure and patient groups in which the described "internal brace" principle will lead to a satisfactory combination of curve correction, preserved instrumentation stability, and maximum spinal growth.

\section{REFERENCES}

1. Vitale MG, Matsumoto H, Bye MR, et al. A retrospective cohort study of pulmonary function, radiographic measures, and quality of life in children with congenital scoliosis: an evaluation of patient outcomes after early spinal fusion. Spine. 2008;33:1242-1249.

2. Dubousset J, Herring JA, Shufflebarger H. The crankshaft phenomenon. J Pediatr Orthop. 1989;9:541-550.

3. McCarthy RE, Sucato D, Turner JL, Zhang H, Henson MA, McCarthy K. Shilla growing rods in a caprine animal model: a pilot study. Clin Orthop Relat Res. 2010;468:705-710.

4. Ouellet J. Surgical technique: modern Luque trolley, a self-growing rod technique. Clin Orthop Relat Res. 2011;469:1356-1367.

5. Luque ER. Segmental spinal instrumentation for correction of scoliosis. Clin Orthop Relat Res. 1982:192-198.

6. Bernard TN Jr, Johnston CE II, Roberts JM, Burke SW. Late complications due to wire breakage in segmental spinal instrumentation. Report of two cases. J Bone Joint Surg Am. 1983;65:1339-1345.

7. Cervellati S, Bettini N, Bianco T, Parisini P. Neurological complications in segmental spinal instrumentation: analysis of 750 patients. Eur Spine J. 1996;5:161-166.

8. Geremia GK, Kim KS, Cerullo L, Calenoff L. Complications of sublaminar wiring. Surg Neurol. 1985;23:629-635.

9. Diab M, Smith AR, Kuklo TR, Spinal Deformity Study G. Neural complications in the surgical treatment of adolescent idiopathic scoliosis. Spine. 2007;32:2759-2763.

10. Reames DL, Smith JS, Fu KM, et al. Complications in the surgical treatment of 19,360 cases of pediatric scoliosis: a review of the Scoliosis Research Society Morbidity and Mortality database. Spine. 2011;36:1484-1491.

11. Wilber RG, Thompson GH, Shaffer JW, Brown RH, Nash CL Jr. Postoperative neurological deficits in segmental spinal instrumentation. A study using spinal cord monitoring. $J$ Bone Joint Surg Am. 1984;66:1178-1187.

12. Eysel P. [Biomechanical principles of ventral and dorsal instrumentation correction in scoliosis]. Orthopade. 2000;29:507-517.

13. Yazar T, Gurkan I, Yilmaz C. A new approach to scoliosis. Eur Spine J. 1999;8:86-92.

14. Boeree NR, Dove J. The selection of wires for sublaminar fixation. Spine. 1993;18:497-503.

15. Nectoux E, Giacomelli MC, Karger C, Herbaux B, Clavert JM. Complications of the Luque-Galveston scoliosis 
correction technique in paediatric cerebral palsy. Orthop Traumatol Surg Res. 2010;96:354-361.

16. Thompson GH, Lenke LG, Akbarnia BA, McCarthy RE, Campbell RM Jr. Early onset scoliosis: future directions. $J$ Bone Jount Surg Am. 2007;89(suppl 1):163-166.

17. Songer MN, Spencer DL, Meyer PR Jr, Jayaraman G. The use of sublaminar cables to replace Luque wires. Spine. 1991;16:S418-S421.

18. Weiss HR. Is there a body of evidence for the treatment of patients with adolescent idiopathic scoliosis (AIS)? Scoliosis. 2007;2:19.

19. Parsons JR, Chokshi BV, Lee CK, Gundlapalli RV, Stamer D. The biomechanical analysis of sublaminar wires and cables using luque segmental spinal instrumentation. Spine. 1997;22:267-273.

20. Mardjetko SM, Hammerberg KW, Lubicky JP, Fister JS. The Luque trolley revisited. Review of nine cases requiring revision. Spine. 1992;17:582-589.

21. Pratt RK, Webb JK, Burwell RG, Cummings SL. Luque trolley and convex epiphysiodesis in the management of infantile and juvenile idiopathic scoliosis. Spine. 1999;24:1538-1547.

22. Goldenberg Y, Tee JW, Salinas-La Rosa CM, Murphy M. Spinal metallosis: a systematic review. Eur Spine J. 2016;25:1467-1473.

23. Lukina E, Laka A, Kollerov $M$, et al. Metal concentrations in the blood and tissues after implantation of titanium growth guidance sliding instrumentation. Spine J. 2016;16:380-388.

24. Akbarnia BA, Cheung $\mathrm{K}$, Noordeen $\mathrm{H}$, et al. Next generation of growth-sparing techniques: preliminary clinical results of a magnetically controlled growing rod in 14 patients with early-onset scoliosis. Spine (Phila Pa 1976). 2013;38:665670 .

25. Dannawi Z, Altaf F, Harshavardhana NS, El Sebaie H, Noordeen H. Early results of a remotely-operated magnetic growth rod in early-onset scoliosis. Bone Joint J. 2013;95-B:7580 .
26. Teoh KH, Winson DM, James SH, et al. Do magnetic growing rods have lower complication rates compared with conventional growing rods? Spine J. 2016;16:S40-S44.

27. Joyce TJ, Smith SL, Rushton PR, et al. Analysis of explanted magnetically controlled growing rods from seven UK spinal centers. Spine. 2018;43:E16-E22.

28. Mazda K, Ilharreborde B, Even J, Lefevre Y, Fitoussi F, Pennecot GF. Efficacy and safety of posteromedial translation for correction of thoracic curves in adolescent idiopathic scoliosis using a new connection to the spine: the Universal Clamp. Eur Spine J. 2009;18:158-169.

29. Bogie R, Roth A, Faber S, et al. Novel radiopaque UHMWPE sublaminar wires in a growth-guidance system for the treatment of early onset scoliosis: feasibility in a large animal study. Spine. 2014;39(25):E1503-E1509.

30. Takahata M, Ito M, Abumi K, et al. Comparison of novel ultra-high molecular weight polyethylene tape versus conventional metal wire for sublaminar segmental fixation in the treatment of adolescent idiopathic scoliosis. J Spinal Disord Tech. 2007;20:449-455.

Corresponding Author: Rob Bogie, MD, Department of Orthopaedic Surgery, Maastricht University Medical Centre, PO Box 5800, NL-6202 AZ Maastricht, The Netherlands. Phone: +31-433875037; Fax: +31-43-3874893; Email: robbogie@ hotmail.com.

Published 30 April 2020

This manuscript is generously published free of charge by ISASS, the International Society for the Advancement of Spine Surgery. Copyright (C) 2020 ISASS. To see more or order reprints or permissions, see http://ijssurgery.com. 